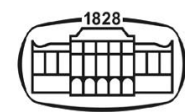

AKADÉMIAI KIADÓ

Acta Microbiologica et Immunologica Hungarica

67 (2020) 1, 49-55

DOI: $10.1556 / 030.66 .2019 .030$

(c) 2019 Akadémiai Kiadó, Budapest

ORIGINAL ARTICLE

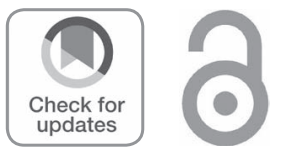

\title{
Prevalence of carbapenem-hydrolyzing OXA-type $\beta$-lactamases among Acinetobacter baumannii in patients with severe urinary tract infection
}

\author{
SMILINE AS GIRIJA ${ }^{1 *}$, JAYASEELAN VIJAYASHREE \\ PRIYADHARSINI $^{2}$ and ARUMUGAM PARAMASIVAM ${ }^{2}$
}

\footnotetext{
${ }^{1}$ Department of Microbiology, Saveetha Dental College and Hospitals, Saveetha Institute of Medical and Technical Sciences (SIMATS), Chennai, India

${ }^{2}$ BRULAC-DRC, Saveetha Dental College and Hospitals, Saveetha Institute of Medical and Technical Sciences (SIMATS), Chennai, India
}

Received: August 16, 2019 • Accepted: September 02, 2019 • Published online: December 09, 2019

\begin{abstract}
Acinetobacter baumannii produces carbapenemase-hydrolyzing class D $\beta$-lactamases (CHDLs) as one of the major drug resistance mechanisms. This investigation is thus aimed to assess the prevalence and to characterize the CHDL-producing strains of $A$. baumannii by both phenotypic assays and genotypic characterization. A total of 73 isolates of A. baumannii were phenotypically and genotypically characterized from patients $(N=1,000)$ with severe urinary tract infection. Tested strains were subjected to double disk synergy testing by Kirby-Bauer disk diffusion method with modified Hodge test (MHT) for carbapenemase production. Plasmid DNA was molecularly screened for CHDL-encoding $b l a_{\text {oxa-51 }}$, $b l a_{\text {oxa-23 }}$, and $b l a_{\text {oxa-143 }}$ genes by polymerase chain reaction. Carbapenem-resistant profile showed $100 \%$, $61.64 \%$, and $67.12 \%$ resistance by Kirby-Bauer disk diffusion method that correlated with MHT positivity for $100 \%(n=73), 80 \%(n=36)$, and $78 \%(n=38)$ of the isolates against imipenem, doripenem, and meropenem, respectively. The $b l a_{\text {oxa-51 }}$ and $b l a_{\text {oxa-23 }}$ were observed in $41.09 \%$ $(n=30)$ and $35.61 \%(n=26)$ with co-occurrence in $4.10 \%(n=3)$ of the isolates. MHT-positive isolates showed $100 \%, 91.66 \%$, and $71.4 \%$ for $b l a_{\text {oxa-51 }}$ and $91.78 \%, 51.11 \%$, and $34.69 \%$ for $b l a_{\text {oxa-23 }}$ with imipenem, doripenem, and meropenem resistance, respectively. None of the strains yielded $b l a_{\text {oxa- } 143}$ gene. The findings of this study showed prevalence of carbapenem resistance and high frequency of $b l a_{\text {oxa-51 }}$ and bla $a_{\text {oxa-23 }}$ among A. baumannii.
\end{abstract}

\section{KEYWORDS}

Acinetobacter baumannii, carbapenems, $b / a_{\text {oxa-51 }}, b / a_{\text {oxa-23 }}, b / a_{\text {oxa-143 }}$

\section{* Corresponding author: Dr. Smiline AS Girija Department of Microbiology, Saveetha Dental College and Hospitals, Saveetha Institute of Medical and Technical Sciences (SIMATS), 162, Poonamallee High Road, Velappanchavadi, Chennai 600077 , Tamil Nadu, India \\ Phone: +91 4426801571 \\ E-mail: smilinejames25@gmail.com}

\section{INTRODUCTION}

Acinetobacter baumannii is an important nosocomial pathogen associated with recalcitrant urinary tract infections, septicemia and pneumonia, and is considered as a frequent cause of infections among patients in intensive care units (ICUs) [1]. In recent years, it is of major concern that $A$. baumannii exhibits multidrug resistance against the routine drugs of choice $[2,3]$. A. baumannii infections are alarming with greater concern due to their dramatic rise in the carbapenem resistance pattern and are considered as sentinels of drug resistance with the designation as carbapenem-resistant A. baumannii (CRAB) [4]. Resistance to carbapenems is mainly mediated by carbapenemases through different classes of genetic determinants [5]. Metallo- $\beta$-lactamases (MBLs) are rare among these species but prevalence of MBLs was reported as $53.4 \%$ in our earlier studies [6]. However, major contribution for carbapenem resistance was induced through the action of carbapenem-hydrolyzing class $\mathrm{D} \beta$-lactamases 
(CHDLs), which are also referred as oxacillinases that can cause mild hydrolysis of the administered carbapenems in patients $[7,8]$ and are often overexpressed in association with insertion sequences [9].

At present, oxacillinases are encoded by five different subclasses of $b l a_{\text {oxa }}$ in $A$. baumannii strains. The $b l a_{\text {oxa-51 }}$ is documented to be associated with intrinsic resistant with 70 variants. Few acquired genes are also reported namely, $b l a_{\text {oxa-23-like, }} b l a_{\text {oxa-24-like, }} b l a_{\text {oxa-58-like, }}$ and $b l a_{\text {oxa-143-like genetic }}$ determinants encoded by both chromosomes and plasmids [10]. Basically, oxacillinases are considered as unusual $\beta$-lactamases forming a heterogenous group based on structural and biochemical properties with a potent hydrolyzing effect on oxacillin than benzyl penicillin. They are also known to hydrolyze amoxicillin, methicillin, cephaloridine, and to some extent cephalothin. Hydrolytic efficiency of carbapenemase hydrolyzing class D $\beta$-lactamase (CHDL) is $100-1,000$-fold lower compared to that of MBL; however, it plays a role in inducing carbapenem resistance and still is frequently reported in A. baumannii [11]. Although MBLs are considered to be more potent than CHDLs, oxacillinases are known to hydrolyze imipenem but not always meropenem [12].

In addition, CHDL-producing $A$. baumannii often exhibits resistance against clavulanate and tazobactam, with susceptibility to $\mathrm{NaCl}$ inhibition, which aids in the laboratory investigations. Among several phenotypic detections, Clinical Laboratory Standards Institute, CLSI guidelines, 2012, advocates the application of modified Hodge test (MHT), CarbaNP test, and/or a molecular based assay for the confirmation of the CHDL producers among Enterobacteriaceae and A. baumannii strains [13]. Genotypic characterization of CHDL-producing strains is based on the detection of genetic determinant $b l a_{\text {oxa }}$, that is usually performed by polymerase chain reaction (PCR) and clonal relatedness can be analyzed by various molecular methods [14]. Periodic surveillance on the CHDL-producing A. baumannii would definitely aid in the eradication of the carbapenem-resistant strains in hospitalized patients.

With this background, the present investigation is aimed to phenotypically and genotypically characterize the CHDL producers among $A$. baumannii strains with the phylogenetic assessment of CHDL-based genetic determinants namely, $b l a_{\text {oxa-51 }}, b l a_{\text {oxa-23, }}$ and $b l a_{\text {oxa-143 }}$ screened from the patients with severe urinary tract infections from South India.

\section{MATERIALS AND METHODS}

\section{Study design}

A total of 73 consecutive, non-repetitive A. baumannii isolates that were isolated and identified for a period of 12 months (2014-2015) were phenotypically and genotypically characterized from urine samples of patients with severe urinary tract infections $(N=1,000)$. Severe urinary tract infection was defined in patients with one or more symptoms of frequency or urgency in urination, suprapubic pain, dysuria, and flank pain. Study cases included the outpatients
(OP cases), inpatients (IP cases), and hospitalized patients in ICUs (ICU patients). Proper ethical guidelines and informed consents were obtained prior to beginning of the study. The strains were phenotypically and genotypically confirmed by conventional microbiological analytical tests and PCR, respectively. These characterized strains were subjected to antibiotic susceptibility test by standard Kirby-Bauer disk diffusion method using imipenem $(10 \mu \mathrm{g})$, doripenem $(10 \mu \mathrm{g})$, and meropenem $(10 \mu \mathrm{g})$ for the carbapenemresistant profile of the selected strains under study [15].

\section{Phenotypic confirmatory test}

Detection of CHDL-based oxacillinases or carbapenemases was carried out by MHT. Briefly, 0.5 McFarland standard turbid Escherichia coli ATCC 25922 broth suspensions was lawn cultured on a sterile Mueller-Hinton agar plate. Using a sterile forceps, imipenem $(10 \mu \mathrm{g})$ disk (HiMedia laboratories, Mumbai, India) was placed at the center of the plate and the overnight fresh suspension of A. baumannii test strain was streaked from the center to the periphery of the plate. Based on the CLSI guidelines, a distorted zone after overnight incubation is interpreted as positive for carbapenemase production among members of Enterobacteriaceae. Although it is not recommended for non-fermenting Gram-negative bacilli, the test is conducted as many previous studies have suggested the test to detect CHDLs among A. baumannii strains [16, 17].

\section{Molecular detection of $b l a_{\text {oxa-51 }}, b l a_{\text {oxa-23 }}$, and $b_{\text {oxa-143 }}$ genetic determinants in CHDL producers}

Extraction of plasmid DNA and PCR amplification. All the strains were stored at $-80{ }^{\circ} \mathrm{C}$ in $80 \% / 20 \%(\mathrm{v} / \mathrm{v})$ glycerol in Luria-Bertani medium for genetic stability of resistance upon storage [18]. Plasmid DNA was extracted from fresh cultures of A. baumannii using Qiagen extraction kit in accordance with the manufacturer's instructions and was stored in $-20{ }^{\circ} \mathrm{C}$ until further use. An amount of $15 \mu \mathrm{l}$ of amplification reaction mixtures was prepared by mixing $7.8 \mu \mathrm{l}$ of $2 \times$ Master Mix (Takara, Japan) in $5.6 \mu \mathrm{l}$ of double distilled water. Specific forward and reverse primers (Eurofins Genomic India Pvt. Ltd., Bangalore, India) of $b l a_{\text {oxa-51 }}, b l a_{\text {oxa-23, }}$ and $b l a_{\text {oxa-143 }}$ were added with the standard PCR conditions (Table I). PCR amplification was carried out and the resulting PCR amplicons were examined in 1\% agarose gel electrophoresis containing ethidium bromide, which was visualized in a gel documentation system. The 100-bp DNA ladder was used to confirm the amplicon size.

\section{RESULTS}

Preliminary screening for the carbapenem resistance tested showed $100 \%, 61.64 \%$, and $67.12 \%$ resistance against imipenem, doripenem, and meropenem, respectively, as per CLSI zone interpretative criteria. MHT was positive in $100 \%$ of imipenem-resistant isolates followed by $80 \%(n=36)$ and 
Table I. Primer sequence and PCR conditions to detect bla $\mathrm{OXA}_{51}, b_{\mathrm{OXA}-23}$, and bla $\mathrm{OXA}_{-143}$ among $\mathrm{CHDL}$ producer $A$. baumannii

\begin{tabular}{|c|c|c|c|c|}
\hline Target gene & Primers & Sequence $\left(5^{\prime}-3^{\prime}\right)$ & Annealing temperature $\left({ }^{\circ} \mathrm{C}\right)$ & Amplicon size (bp) \\
\hline \multirow[t]{2}{*}{ bla $_{0 \times A-23-\text { like }}$} & OXA-23-F & GATCGGATTGGAGAACCAGA & \multirow[t]{2}{*}{52} & \multirow[t]{2}{*}{501} \\
\hline & OXA-23-R & АTTTCTGACCGCATTTCCAT & & \\
\hline \multirow[t]{2}{*}{$b a_{0 \times A-51-l i k e}$} & OXA-51-F & TAATGCTTTGATCGGCCTTG & \multirow[t]{2}{*}{52} & \multirow[t]{2}{*}{353} \\
\hline & OXA-51-R & TGGATTGCACTTCATCTTGG & & \\
\hline \multirow[t]{2}{*}{ bla ${ }_{\text {OXA-143-like }}$} & OXA-143-F & TGGCACTTCAGCAGTTCCT & \multirow[t]{2}{*}{52} & \multirow[t]{2}{*}{149} \\
\hline & OXA-143-R & TAATCTTGAGGGGGCCAACC & & \\
\hline
\end{tabular}

Note: PCR: polymerase chain reaction; CHDL: carbapenemase-hydrolyzing class D $\beta$-lactamase; F: forward; R: reverse.

$78 \%(n=38)$ among doripenem- and meropenem-resistant strains (Table II).

Genotypic characterization of the CHDL genetic determinants showed the presence of $b l a_{\text {oxa-51 }}$ and $b l a_{\text {oxa-23 }}$ in $41.09 \% \quad(n=30)$ and $35.61 \% \quad(n=26)$ of the isolates (Figures 1-3). Co-occurrence of bla $a_{\text {oxa-51 }}$ and bla $a_{\text {oxa-23 }}$ was observed in $4.10 \%(n=3)$ of the isolates. MHT-positive isolates showed $100 \%$ positive for $b l a_{\text {oxa-51 }}$ with imipenem resistance, $91.66 \%(n=33)$ with doripenem resistance, and $71.4 \% \quad(n=35)$ with meropenem resistance. Similarly, $b l a_{\text {oxa-23 }}$ was positive in $91.78 \%(n=67)$ with imipenem resistance, $51.11 \%(n=23)$ with doripenem resistance, and $34.69 \%(n=17)$ with meropenem resistance among MHTpositive isolates. Among the three isolates with both $b l a_{\text {oxa-51 }}$ and $b l a_{\text {oxa-23 }}$ genes, only one strain was MHT-positive. However, none of the strains yielded $b l a_{\text {oxa-143 }}$ gene.

\section{DISCUSSION}

CRAB strains were declared as the priority number one pathogen by WHO in the year 2017 [19], due to a wide

Table II. Frequency of CHDL-producing $A$. baumannii based on phenotypic and genotypic characterization assays

\begin{tabular}{|c|c|c|c|c|c|c|}
\hline \multirow[b]{2}{*}{$\begin{array}{l}\text { Isolate under } \\
\text { study }\end{array}$} & \multicolumn{2}{|c|}{ Kirby-Bauer method } & \multirow[b]{2}{*}{ MHT positivity (\%) } & \multicolumn{3}{|c|}{ Genes of target } \\
\hline & $\begin{array}{l}\text { Carbapenems } \\
\text { tested }\end{array}$ & Resistance (\%) & & bla $_{\text {OXA-51 }}(\%)$ & bla $_{\text {OXA-23 }}(\%)$ & bla $_{\text {OXA-143 }}(\%)$ \\
\hline \multirow{3}{*}{$\begin{array}{l}\text { A. baumannii } \\
(N=73)\end{array}$} & Imipenem & 100 & 100 & 100 & 91.78 & 0 \\
\hline & Doripenem & 61.64 & 80 & 91.66 & 51.11 & 0 \\
\hline & Meropenem & 67.12 & 78 & 71.4 & 34.69 & 0 \\
\hline
\end{tabular}

Note: MHT: modified Hodge test; CHDL: carbapenemase-hydrolyzing class D $\beta$-lactamase.

Figure 1. (a) Electrophoretogram of bla ${ }_{0 x a-51}$ gene run along with 100-bp DNA ladder. (b) Electrophoretogram of bla $a_{\text {oxa-23 }}$ amplicons run along with 100-bp DNA ladder

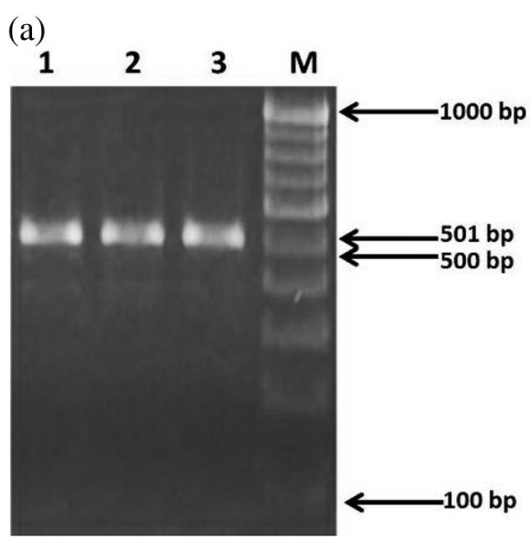

(b)

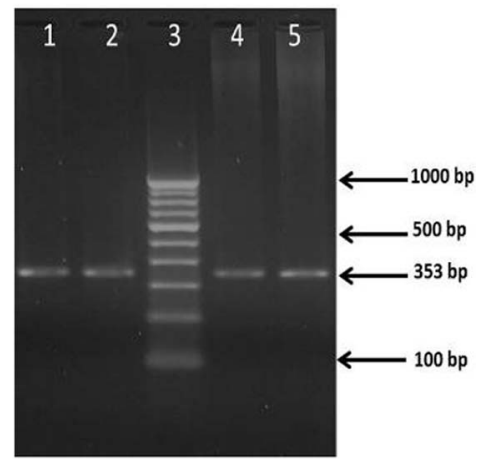


Figure 2. (a) The partial sequence chromatogram of $b a_{0 x a-51}$ gene. (b) The partial sequence chromatogram of bla $a_{0 x a-23}$ gene

(a)

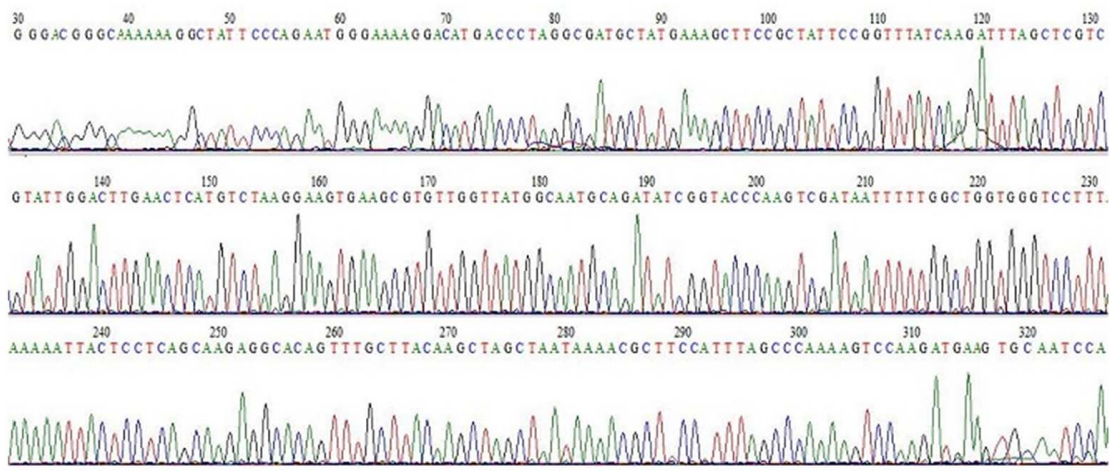

(b)

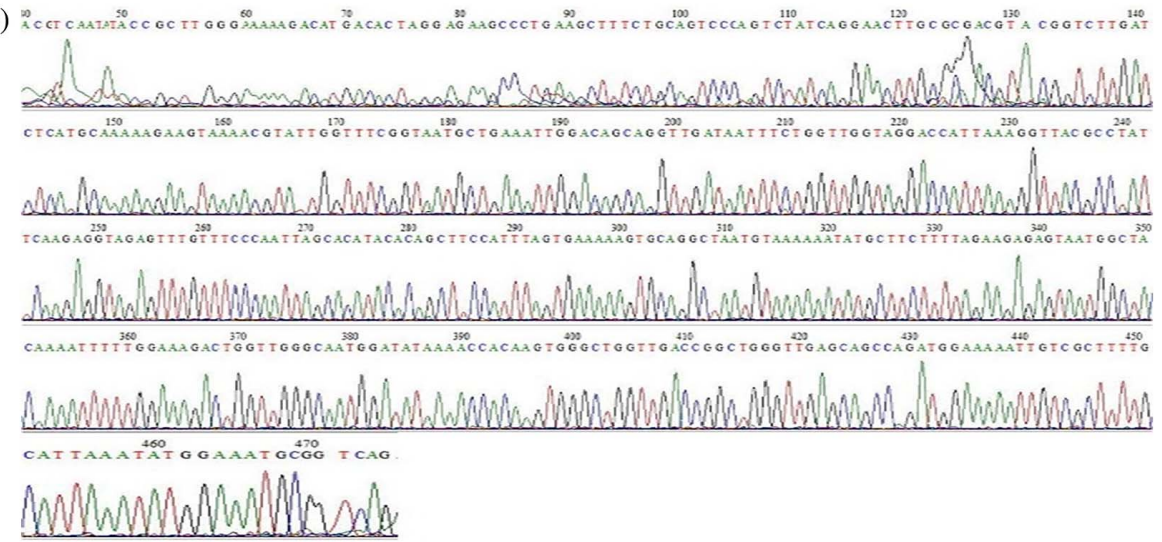

range of nosocomial infections resulted from the strains, encompassing meningitis, septicemia, pneumonia, skin, and wound infections with a major challenge in the patient health care [20]. In addition, severe and complicated infections of A. baumannii are treated with the last resort of carbapenems, such as imipenem, doripenem, meropenem, and ertapenem. High incidences of carbapenem-resistant strains in both community- and hospital-acquired infections have been documented [21]. The present investigation has also recorded $50.68 \%(n=37)$ as carbapenem-resistant strains showing resistance against all the three drugs tested under the study. Hundred percent of the strains showing imipenem resistance in this study correlate with an earlier study from South India [22]. Resistance to imipenem in A. baumannii is reported [23] and in many earlier studies the isolates of A. baumannii for carbapenemase and MBL production were categorized based on imipenem susceptibility and resistance patterns [24]. Higher incidences of imipenem resistance are also documented in various studies globally [6, 25]. Our clinical strains had previously recorded $60 \%-65 \%$ of nonsusceptibility against doripenem and meropenem with only $15.06 \%$ and $13.69 \%$ susceptibility, respectively, against the same [20] that had correlated with similar observations from Turkey with $66.6 \%$ resistance against meropenem and $49.9 \%$ against doripenem mediated by OXA-type carbapenemases [21]. Similar correlations were also observed from a study in the USA that showed $68 \%$ and $80 \%$ non-susceptibility to meropenem and doripenem, respectively [26]. On the contrary, a study from Punjab, India, has recorded only $6 \%$ of the isolates to exhibit non-susceptibility against doripenem and meropenem [22]. Among the routine carbapenems, it is stated that there is no impact in the susceptibility patterns of imipenem, which aids in the reduced administration of imipenem and ciprofloxacin [27]. However, this study has its own limitation where ertapenem is thus omitted under carbapenem-resistant profile for the test organisms under the study.

Phenotypic detection of CHDL production was observed using MHT in this study. Among the tested isolates, with $100 \%$ resistance against imipenem and nearly $63 \%$ resistance against doripenem and meropenem, phenotypic confirmation was achieved in all the imipenem-resistant isolates but only in 36 and 38 isolates of doripenem- and meropenemresistant isolates. Among the 73 imipenem-resistant isolates, all were positive for MHT, which might be due to the $b l a_{\text {oxa-51 }}$ intrinsic gene cassettes associated with integrons [28]. It might also be an additional fact for the $91.78 \%$ and $71.4 \%$ of the isolates showing MHT-positive A. baumannii, together with the expression of $b l a_{\text {oxa-23 }}$, suggesting the role of bla $a_{\text {oxa-51- }}$ and $b l a_{\text {oxa-23-type CHDL's in inducing carba- }}$ penem resistance. Isolates with positive MHT but showing negative genotypic results may be related to the variants exhibited among class I integron structures, which are detected frequently among A. baumannii $[29,30]$. Comparative analysis between phenotypic and genotypic data observed in the present investigation suggests MHT to be highly reliable and easy to perform for the preliminary screening of CHDL production in accordance with earlier reports [31]. 
Figure 3. (a) Multiple sequence alignment of bla ${ }_{\text {oxa-51 }}$ gene using plasmid DNA as the template isolated from A. baumannii. (b) Multiple sequence alignment of $b a_{\text {oxa-23 }}$ gene using plasmid DNA as the template isolated from $A$. baumannii

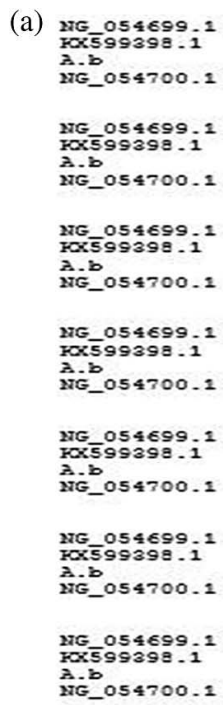

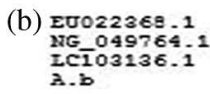

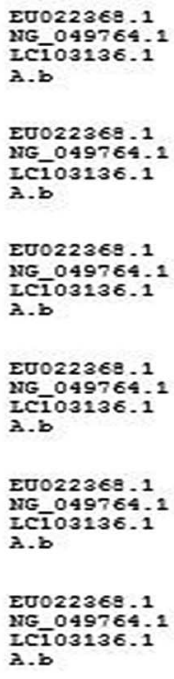

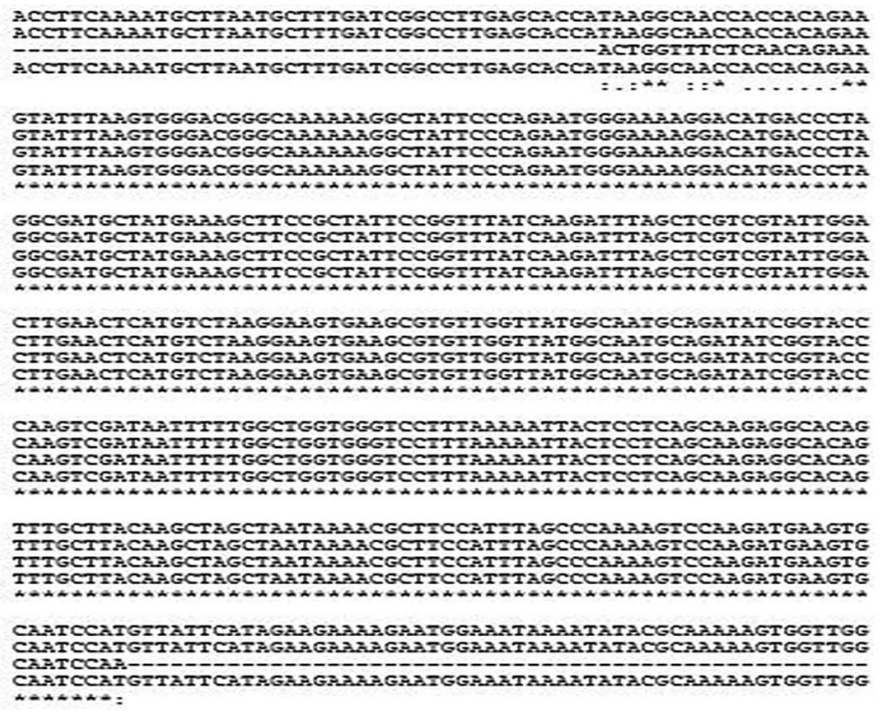

GACACTAGGAGAAGCCATGAAGCTITCTGCAGTCCCAGTCTATCAGgaACTIGCGCGACG GACACTAGGAGAAGCCATGAAGCTITCTGCAGTCCCAGTCTATCAGGAACTIGCGCGAC GACACTAGGAGAGCCCTGAGCTITCTGCAGTCCCAGTCTATCAGGAACTIECGCGAC

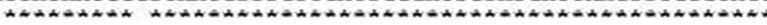
TATCGGTCTIGATCTCATGCAAAAAGAAGIAAAACGTATIGGTITCGTARTGCTGARAI

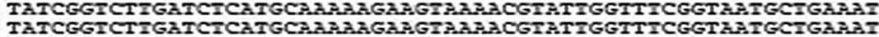
TA-CGGICTIGATCTCATGCAAAAAGAAGTAAAACGTATTGGTTPCGGTAMTGCTGAAAI

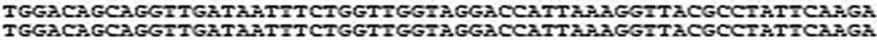
TGGACAGCAGGTTGATAATITCTGGTTGGIAGGACCATTAAAGGITACGCCTATICAAGA TGGACAGCAGGTTGATATTTCTGGTTGGIAGGACCATTAAAGGITACGCCTATICAAGA

GGTAGAGTTRTTTCCCAATTAGCACATACACAGCTICCATITAGTGAARAGTGCAGGC GGTAGAGTTIGTTCCCCAITAGCACATACACAGCTICCATTTAGTGAAAAGTGCAGGC GGTAGAGTTIGTTTCCCAATTAGCACATACACAGCTICCATTTAGTGAAAAAGTGCAGGC

TAATGTAAAAATATGCTTCTITTAGAAGAGATAATGGCTACAAAATTITIGGAAGAC

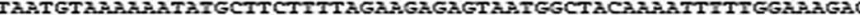
TAATGTAMAAA.ATATGCTTCTITTAGAAGAGAGTAMTGGCTACARA.ATTITIGGAMAGAC TGGTIGGGCAATGGATATAAAACCACAAGIGGGCIGGTIGACCGGCTGGGTIGAGCAGCC

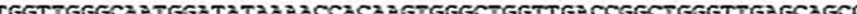
TGGTIGGGCAATGGATATAAAACCACAAGTGGGCTGGTTGACCGGCTGGGTIGAGCAGCC AGRGGAAAATTGICGCTITICCATMAATATGGAAAGCGGTCAGAATGCCGGCATC

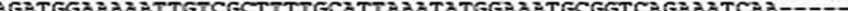
AGATGGAAAARTGICGCTITECATTAARTATGGAAATGCGGTCAGAAA-ATCAAT--
Molecular detection of the genetic determinants of CHDL production namely, $b l a_{\text {oxa-23 }}, b l a_{\text {oxa-51 }}$, and $b l a_{\text {oxa-143, was }}$ observed using PCR. All the resistant isolates $(n=73)$ of $A$. baumannii showed $b l a_{\text {oxa-143 }}$ negativity. In comparison with the carbapenem-resistant profile (IMP - 100\%, Dor 61.64\%, Mero - 67.12\%) and MHT-positive isolates, only 23 and 17 showed the presence of $b l a_{\text {oxa-23. This variation }}$ might be due to the other non-enzymatic mechanisms, such as presence of efflux pumps, role of outer membrane proteins, etc., exhibiting the carbapenem-resistance property among A. baumannii [32], which is the vital fact for the widespread distribution of CHDL producers among $A$. baumannii observed worldwide [33, 34].

Among the CHDL genetic determinants, co-occurrences of the genes are also not uncommon. Studies record the different patterns of co-occurring CHDL genes from different countries including India [35]. In view with this, this study also records the co-occurrence of $b l a_{\text {oxa-23 }}$ and $b l a_{\text {oxa-51, }}$ in three isolates. Comparative analysis between phenotypic and genotypic detection also shows a significant report. The study also records isolates with $\mathrm{MHT}+b l a_{\mathrm{oxa}-23}$ and $\mathrm{MHT}+b l a_{\mathrm{oxa}-51}$ positivity, respectively, with isolates showing $\mathrm{MHT}+b l a_{\text {oxa-23 }}+b l a_{\text {oxa-51 }}$ positivity. In an earlier study from Nepal, the coexistence of $b l a_{\text {oxa-23 }}$ and $b l a_{\mathrm{NDM}-1}$ was detected [36] with the presence of other class B MBLs, such as $b l a_{\mathrm{VIM}}$ and $b l a_{\mathrm{GIM}}$. These reports suggest that the variations exhibited by the test isolates in both phenotypic and genotypic characterizations are mainly due to the frequency of different genetic determinants prevailing among the A. baumannii species existing in different geographical location against the carbapenems.

Complications induced by $A$. baumannii traits that are acquired through different patterns of antimicrobial 
resistance transform them as dreadful nosocomial pathogen posing serious impediments in infection control. Frequency of CHDLs and the distribution of their genetic determinants restrict the administration of carbapenems against A. baumannii. The present investigation thus concludes by stating the need for the proper and periodical antimicrobial surveillance programs for the use of carbapenems against A. baumannii due to the high prevalence of varying resistance pattern in association with the $b l a_{\text {oxa-23 }}, b l a_{\text {oxa-51 }}$, and $b l a_{\text {oxa-143 }}$ in inducing the carbapenemase resistance.

Conflict of Interest: The authors declare no conflict of interest.

\section{REFERENCES}

1. Berezin, E. B., Towner, K. J.: Acinetobacter spp. as nosocomial pathogens: Microbiological, clinical, and epidemiological features. Clin Microbiol Rev 9, 148-165 (1996).

2. Talbot, G. H., Bradley, J., Edwards, J. E., Gilbert, D.: Bad bugs need drugs: An update on the development pipeline from the Antimicrobial Availability Task Force of the Infectious Diseases Society of America. Clin Infect Dis 42, 657-668 (2006).

3. Dijkshoorn, L., Nemec, A., Seifert, H.: An increasing threat in hospitals: Multidrug-resistant Acinetobacter baumannii. Nat Rev Microbiol 5, 939-951 (2007).

4. Richet, H. M., Mohammed, J., McDonald, L. C.: Building communication networks: International network for the study and prevention of emerging antimicrobial resistance. Emerg Infect Dis 7, 319-322 (2000).

5. Poirel, L., Bonnin, R. A., Nordmann, P.: Genetic basis of antibiotic resistance in pathogenic Acinetobacter species. IUBMB Life 63, 1061-1067 (2011).

6. Smiline Girija, A. S., Vijayashree Priyadharsini, J., Paramasivam, A.: Prevalence of VIM and GIM producing Acinetobacter baumannii from patients with severe UTI. Acta Microbiol Immunol Hung 65, 539-550 (2018).

7. Higgins, P. G., Dammhayn, C., Hackel, M., Seifert, H.: Global spread of carbapenem-resistant Acinetobacter baumannii. J Antimicrob Chemother 65, 233-238 (2010).

8. Poirel, L., Naas, T., Nordmann, P.: Diversity, epidemiology, and genetics of class D $\beta$-lactamases. Antimicrob Agents Chemother 54, 24-38 (2010).

9. Woodford, N., Ellington, M. J., Coelho, J. M., Turton, J. F.: Multiplex PCR for genes encoding prevalent OXA carbapenemases in Acinetobacter spp. Int J Antimicrob Agents 27, 351-353 (2006).

10. Higgins, P. G., Lehmann, M., Seifert, H.: Inclusion of OXA-143 primers in a multiplex polymerase chain reaction (PCR) for genes encoding prevalent OXA carbapenemases in Acinetobacter spp. Int J Antimicrob Agents 35, 305-312 (2010).

11. Poirel, L., Nordmann, P.: Carbapenem resistance in Acinetobacter baumannii: Mechanisms and epidemiology. Clin Microbiol Infect 12, 826-836 (2006).

12. Nordmann, P., Poirel, L.: Emerging carbapenemases in Gramnegative aerobes. Clin Microbiol Infect 8, 321-331 (2002).
13. Clinical Laboratory Standards Institute [CLSI]: Performance Standards for Antimicrobial Susceptibility Testing. Table 3A: M02-A12 and M07-A10. CLSI, Wayne, PA, 2012.

14. Srinivasa, V. B., Rajamohan, G., Pancholi, P., Stevenson, K., Tadesse, D., Patchanee, P., Marcon, M., Gebreyes, W. A.: Genetic relatedness and molecular characterization of multidrug resistant Acinetobacter baumannii isolated in central Ohio, USA Gebreyes. Ann Clin Microbiol Antimicrob 8, 21-26 (2009).

15. Smiline Girija, A. S., Vijayashree Priyadharsini, J., Paramasivam, A.: CLSI based antibiogram profile and the detection of MDR and XDR strains of Acinetobacter baumannii isolated from urine samples. Med J Islamic Rep Iran 33, 11-16 (2019).

16. Yang, H. Y., Lee, H. J., Suh, J. T., Lee, K. M.: Outbreaks of imipenem resistant Acinetobacter baumannii producing OXA-23 $\beta$-lactamase in a tertiary care hospital in Korea. Yonsei Med J 50, 764-70 (2009).

17. Andriamanantena, T. S., Ratsima, E., Rakotonirina, H. C., Randrianirina, F., Carod, J. F., Richard, V., Talarmin, A.: Dissemination of multidrug resistant Acinetobacter baumannii in various hospitals of Antananarivo Madagascar. Ann Clin Microbiol Antimicrob 9, 17-23 (2010).

18. Maleki, M. H., Sekawi, Z., Soroush, S., Azizi-Jalilian, F.: Phenotypic and genotypic characteristics of tetracycline resistant Acinetobacter baumannii isolates from nosocomial infections at Tehran hospitals. Iran J Basic Med Sci 17, 21-26 (2014).

19. Tacconelli, E., Magrini, N.: Global Priority List of AntibioticResistant Bacteria to Guide Research, Discovery, and Development of New Antibiotics. Available at http://www.who.int/ medicines/publications/WHO-PPL-Short_Summary_25FebET_NM_WHO.pdf?ua=1

20. Sinha, M., Srinivasa, H.: Mechanisms of resistance to carbapenems in meropenem resistant Acinetobacter isolates from clinical samples. Indian J Med Microbiol 25, 121-125 (2007).

21. Terzi, H. A., Atasoy, A. R., Aykan, S. B., Karakece, E., Asık, G., Ciftci, I. H.: Association of doripenem resistance with OXAtype carbapenemases in Acinetobacter baumannii isolates. Saudi Med J 37, 43-47 (2016).

22. Goyal, K., Gautam, V., Ray, P.: Doripenem vs meropenem against Pseudomonas and Acinetobacter. Indian J Med Microbiol 30, 350-351 (2012).

23. Hussein, H. N., Al-Mathkhury, J. F., Sabbah, A. M.: Imipenem resistant Acinetobacter baumannii isolated from patients and hospitals environment in Baghdad. Iraq J Sci 54, 803-812 (2013).

24. Daef, E. A., Mohamed, I. S., Ahmed, A. S., El-Gendy, S. G.: Detection of outbreak caused by multidrug resistant Acinetobacter baumannii in Assiut University Hospitals. Afr J Microbiol Res 8, 2238-2244 (2014).

25. Taneja, N., Maharwal, S., Sharma, M.: Imipenem resistance in non-fermenters causing nosocomial urinary tract infections. Ind J Med Sci 57, 294-299 (2003).

26. Esterly, J. S., Qi, C., Malczynski, M., Scheetz, M. H.: Predictability of doripenem susceptibility in Acinetobacter baumannii isolates based on other carbapenem susceptibilities and $b l a_{\text {OXA }}$ gene status. Pharmacotherapy 30, 354-360 (2010).

27. Sousa, D., Castelo-Corral, L., Gutiérrez-Urb, J. M., Molina, F., Lopez-Calvi, B.: Impact of ertapenem use on Pseudomonas 
aeruginosa and Acinetobacter baumannii imipenem susceptibility rates: Collateral damage or positive effect on hospital ecology? J Antimicrob Chemother 68, 1917-1925 (2013).

28. Da Silva, G. J., Correia, M., Vita, C., Ribeiro, G.: Molecular characterization of $b l_{\mathrm{IMP}-5}$, a new integron-borne metallo- $\beta$ lactamase gene from an Acinetobacter baumannii nosocomial isolate in Portugal. FEMS Microbiol Lett 215, 33-39 (2002).

29. Ellington, M. J., Kistler, J., Livermore, D. M., Woodford, N.: Multiplex PCR for rapid detection of genes encoding acquired metallo-beta-lactamases. J Antimicrob Chemother 59, 321-322 (2007).

30. Seward, R. J.: Detection of integrons in worldwide nosocomial isolates of Acinetobacter spp. Clin Microbiol Infect 5, 308-318 (1999).

31. Sung, J. Y., Kwon, K. C., Park, J. W., Kim, Y. S.: Dissemination of IMP-1 and OXA type -lactamase in carbapenem-resistant Acinetobacter baumannii. Korean J Lab Med 28, 16-23 (2008).

32. Limansky, A. S., Mussi, M. A., Viale, A. M.: Loss of a 29-kilodalton outer membrane protein in Acinetobacter baumannii is associated with imipenem resistance. J Clin Microbiol 40, 4776-4778 (2002).

33. Afzal-Shah, M., Woodford, N., Livermore, D. M.: Characterization of OXA-25, OXA-26, and OXA-27, molecular class D $\beta$-lactamases associated with carbapenem resistance in clinical isolates of Acinetobacter baumannii. Antimicrob Agents Chemother 45, 583-588 (2001).

34. Bou, G., Oliver, A., Martınez-Beltra, J.: OXA-24, a novel class D $\beta$-lactamase with carbapenemase activity in an Acinetobacter baumannii clinical strain. Antimicrob Agents Chemother 44, 1556-1561 (2000).

35. Amudhan, S. M., Sekar, U., Arunagiri, K., Sekar, B.: OXA beta-lactamase-mediated carbapenem resistance in Acinetobacter baumannii. Ind J Med Microbiol 29, 269-274 (2011).

36. Joshi, P. R., Acharya, M., Kakshapati, T., Leungtongkam, U.: Co-existence of $b l a_{\mathrm{OXA}-23}$ and $b l a_{\mathrm{NDM}-1}$ genes of Acinetobacter baumannii isolated from Nepal: Antimicrobial resistance and clinical significance. Antimicrob Resist Infect Control 6, 21-25 (2017). 